\section{Carotid stenosis due to clamp injury}

We report a case of symptomatic stenosis of the common carotid, which we consider to have been caused by arterial clamping during a previous operation.

\section{Case report}

A 52-year-old hypertensive woman was referred in May 1978 with recurrent transient ischaemic attacks affecting the right arm, usually associated with transient loss of consciousness. Bilateral carotid angiography showed $95 \%$ stenosis at the origin of the left internal carotid artery and an atheromatous plaque at the origin of the right internal carotid artery without appreciable stenosis.

An uneventful left carotid endarterectomy using a Brenner shunt was performed; four weeks later she was well but a loud left carotid bruit persisted. Over the next 16 months she suffered further episodes of loss of consciousness, which were associated with headache but no focal signs. In October 1979 these attacks were accompanied by paraesthesia of the left arm and blurred vision, and a right carotid endarterectomy was performed. Three months later further transient ischaemic attacks affecting the right arm occurred, which were thought to be due to restenosis of the left internal carotid artery and release of emboli, but despite treatment with antiplatelet drugs and then with warfarin her symptoms increased in frequency.

An electroencephalogram and brain scan were normal. Spectral analysis of continuous-wave Doppler signals disclosed turbulence in the left common carotid, but normal sonograms were obtained from the internal and external carotid arteries. An arch aortogram showed an ususual stenotic lesion (figure $(a),(b))$ in the mid-portion of the left common carotid at about the site where an arterial clamp would have been applied at the time of the left carotid endarterectomy. There was no appreciable restenosis at the carotid bifurcations.

The left common carotid was re-explored and found to contain two circumferential intimal flaps about $1 \mathrm{~cm}$ apart (figure $(c)$ ), which considerably obstructed flow. These were separated by a bare fibrous area but there was no adherent thrombus. The intimal flaps were excised and a vein-patch angioplasty performed. Postoperative Doppler studies indicated improved flow in the common carotid, and a sonogram was normal. She made a satisfactory recovery and did not have any further ischaemic attacks.

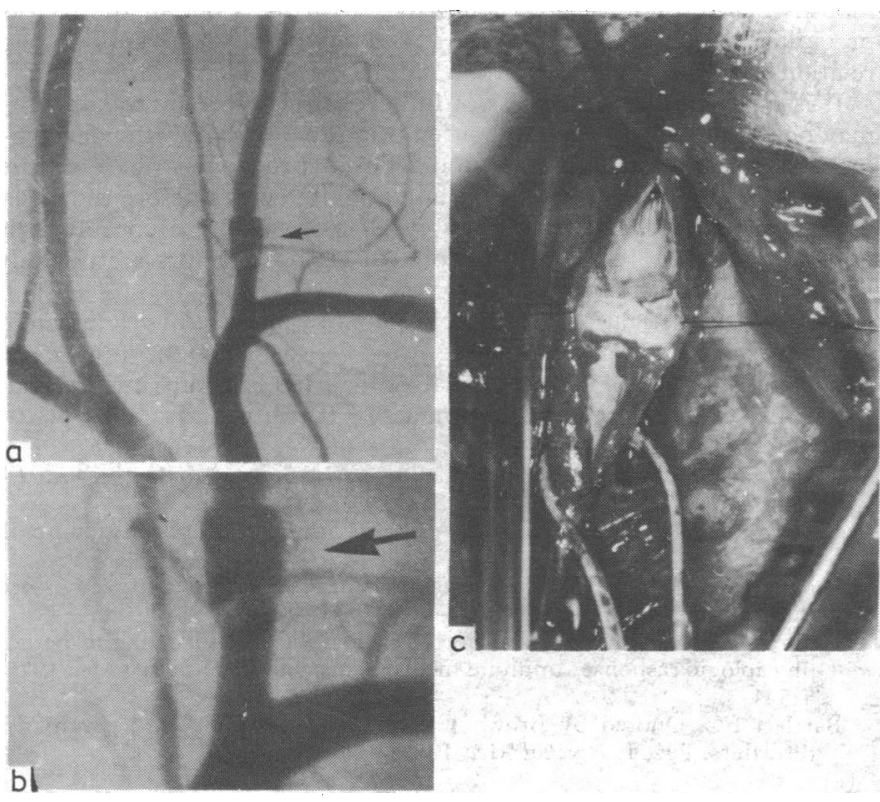

Stenosis of left common carotid on arch aortography (a), with enlarged view (b) showing double stenotic lesion. Double circumferential intimal flaps were found at operation $(c)$.

\section{Comment}

Intimal injury at the site of arterial clamping is not well documented. Henson and Rob ${ }^{1}$ reported a comparative study of the effects of different arterial clamps applied to the gastroepiploic artery during partial gastrectomy and described a case of early occlusion caused by damage by a clamp during repair of a lacerated femoral artery. Slayback $e t a l^{2}$ in a study of various clamps on normal and atherosclerotic rabbit arteries reported that varying degrees of intimal damage were almost a constant finding. Harvey and Gough ${ }^{3}$ studied the effects of five vascular clamps on dog femoral arteries and found that the degree of vessel-wall damage depended on the type of clamp used and the pressure exerted by the jaws of the clamp.

We have been unable to find any references to clamp-induced carotid stenosis. Thompson ${ }^{4}$ did not mention it in his paper on the complications of carotid endarterectomy nor did Callow ${ }^{5}$ in a collective review of the incidence of restenosis after 13470 operations.

We believe that this lesion was caused either by the application of an arterial clamp (deBakey type) before and after shunting or by the pressure of a silk ligature snare used to maintain the position of the Brenner shunt, resulting in an intimal fracture. Perhaps surprisingly, the distal flap did not dissect and produce a carotid occlusion. Fortunately, this appears to be rare, but our case illustrates one of the hazards of arterial compression by clamps and snares.

${ }^{1}$ Henson GF, Rob CG. A comparative study of the effects of differen arterial clamps on the vessel wall. Brf Surg 1956;182:561-4.

2 Slayback JB, Bowen WW, Hinshaw DB. Intimal injury from arteria clamps. Am $\mathcal{F}$ Surg 1976;132:183-8.

${ }^{3}$ Harvey JG, Gough $\mathrm{M}$. The traumatic effect of the vascular clamp. $\mathrm{Br} \mathcal{F}$ Surg $1981 ; 68: 267-72$.

4 Thompson JE. Complications of carotid endarterectomy and their prevention. Worldf Surg 1979;3:155-65.

5 Callow AD. An overview of the stroke problem in the carotid territory. Am F Surg 1980;140:181-91.

(Accepted 13 March 1981)

Department of Surgery, Queen Elizabeth Hospital, Birmingham B15 2TH

A AUKLAND, FRCs, clinical research fellow

R A HURLOW, FRCS, senior lecturer in surgery

\section{Recurrent abdominal pain and lactose intolerance in childhood}

Recurrent abdominal pain is one of the most common ailments seen in paediatric practice, affecting about $10 \%$ of schoolage children. The aetiology of this problem remains ill defined. Two recent studies from the USA, using tests for lactose malabsorption and elimination diets, incriminated lactose intolerance as the cause of the abdominal pain in over $25 \%$ of children. ${ }^{12}$ In contrast, Christensen, in a study of 50 children with recurrent abdominal pain in Denmark, detected only one case fulfilling the criteria of lactose intolerance. ${ }^{3}$ Not surprisingly, the American findings do not have worldwide válidity in view of ethnic and regional variations in the age of onset of lactose malabsorption. The purpose of our study was to establish the incidence of lactose intolerance in Caucasian children with recurrent abdominal pain in the north of England.

\section{Patients, methods, and results}

A lactose-hydrogen breath test was performed in 26 Caucasian children with a history of recurrent abdominal pain (age range 4-14 years, mean 9 years). Recurrent abdominal pain was defined as three or more episodes of unexplained pain severe enough to affect activity and occurring over a period of three months. Before inclusion in the study each child underwent thorough physical examination and full blood count and urine analysis were carried out. In most children an attempt to find an organic cause led to more extensive investigation and included radiological studies.

The hydrogen breath test is a measure of the change in breath hydrogen concentration caused by bacterial fermentation of unabsorbed sugar in the colon after a sugar load. It has the advantage of being non-invasive and more sensitive than the conventional lactose tolerance test, in which blood sampling is required and the results are affected by the rate of stomach emptying.

After an overnight fast the children were given lemon-flavoured lactose $(2 \mathrm{~g} / \mathrm{kg}$; maximum $50 \mathrm{~g})$ as a $20 \%$ aqueous solution. Breath samples wer collected by the nasal prong technique at 30 -minute intervals for two hours. $A$ rise in hydrogen concentration of 10 parts per million above the baseline value at 90 or 120 minutes was regarded as indicating lactose malabsorption. Breath samples were analysed for hydrogen by gas chromatography. No child was taking antibiotics when tested.

A result indicating lactose malabsorption was obtained in three of the 26 $(12 \%)$ children, two of whom experienced pain after ingesting the lactose. In one of these two children a six-week lactose-free diet resulted in an appreciable improvement in symptoms. Thus only one of the 26 children (3.8\%) had the combined features of lactose malabsorption with abdominal pain, which was induced by ingestion of lactose and relieved by withdrawal of it. 